\title{
ANALYSIS STUDENTS' ANXIETY IN LEARNING SPEAKING USING DRILL METHOD
}

\author{
Wina Juwitawati ${ }^{1}$, Andiany Retno Pratiwi ${ }^{2}$ \\ ${ }^{1}$ IKIP Siliwangi \\ ${ }^{2}$ IKIP Siliwangi \\ ㄹwinajuwitawati@gmail.com, $\underline{\text { 2andiyanipratiwi6@gmail.com }}$
}

\begin{abstract}
Speaking skill is one of the most difficult aspects of language learning. Students find it difficult to express themselves in spoken language. This research is to know how far the anxiety students in learning speaking. Anxiety analysis is very important because it will affect the extent to which goals will be achieved. Researchers analyze level of anxiety in learning speaking used drill method and also used FLCAS ( Foreign Language Classroom Anxiety Scale) questionnaire by Howritz et al (1986) for collecting data. The FLCAS data questionnaire consisting of 33 items scoring with Likert's scale and analyzed manually used Oeting's scale. The researchers take 20 students from X mipa 1 of SMA Pasundan Cikalongkulon Cianjur in the academic year 2017/2018. In conclusion the research showed that there is a significant and it is difference indicated that drill method was effective in reduced students' anxiety in learning speaking.
\end{abstract}

Keywords: Speaking, Drill method, Anxiety

\section{INTRODUCTION}

Speaking is important factor in learning. (Wahyuni, 2016) stated that speaking is the ability to produce the language and share their ideas. Other definition about speaking comes from Lado (1961: 240) stated in (Wahyuni, 2016) describes speaking as the ability to express oneself in life situation, or to express a sequence of ideas fluently. It means that speaking is the ability to express the idea and to produce the spoken language. There are some difficulties for speaking skill that teachers can come across in helping students to speak in the classroom.these are inhibition, lack of topical knowledge, low participation, mother-tongue used, and most of students think that speaking is the hardest lesson in learning English, and usually they feel anxious in class.

Anxiety is one of the most well documented psychological phenomena. In general anxiety appears from human body as a response to a particular situation. Commonly anxiety can be identified as a feeling of being threatened, apprehension, tension, or worry. There are several definitions of anxiety which are found. (Carlson and Buskist, 1997) say anxiety is "a sense of apprehension or doom that is accompanied by certain physiological reactions, such as accelerated heart rate, sweaty palms, and tightness in the stomach."

Furthermore, anxiety arises as a response to a particular situation, (Passer et al., 2009) define anxiety as a state of tension and apprehension as a natural response to perceived threat. It means that people especially student are naturally feel anxious when they are threatened. (Ormrod, 


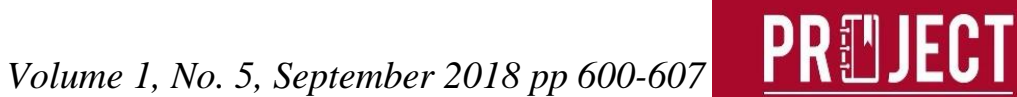

2011) states that anxiety is a feeling of uneasiness and apprehension concerning a situation with an uncertain outcome.

Although anxiety and fear sounds similar, both are actually different. (Halgin and Whitbourne, 2007) describe the difference between fear and anxiety, fear is a natural alarm response to a dangerous situation while anxiety is more future-oriented, a feeling of apprehension and uneasiness about the possibility of something terrible might happen.

It also can be stressful when they are expected to speak in foreign language before the fluency is achieved. In line with the issue of anxiety, (Tornbury, 2005) states that the lack of vocabularies, improper grammar and fears of mistakes are some of the factors that can contribute the failure of speaking English, and cause an acute sense of anxiety when it comes to speaking.

Based on those background, to find out how far levels of anxiety students' inlearning speaking, the researchers feel investigate the anxiety.Teachers have many method in teaching. One of the teaching is drill method. (Kartikasari, Zainal and Salam, 2014) state that drilling is a technique that has been used in the foreign language classroom for many years. (Matthews, Spratt and Nelson, 1991) said that "A drill is a type of highly controlled oral practice in which the students respond to given cue.

The study was conducted on the second semester students in SMA PASUNDAN Cikalong Kulon Cianjur. The concern of this study is investigating anxiety and finding out the strategies. Hopefully, this study can be a solution for the students and the teachers on how to teach and learn speaking effectively, and also as a references for the students who conduct the similar research.

\section{METHOD}

In this research the researchers used qualitative research design. Based on theory (Kaswan \& Suprijadi, 2016), a qualitative research design is a research design that place primary importance on studying small samples of purposely chosen individual. In addition Yin (2011) in (Kaswan \& Suprijadi, 2016) state that qualitative data are more alphabetic than numeric. The data are likely to be represented in narratives or in data arrays, such as the word tables, hierarchies, matrices, and other types of diagrams.

The researchers used FLCAS (Foreign Language Classroom Anxiety Scale) questionnaire develop by Horwitz, et al (1986) stated in (Mayangta, 2013). FLCAS IS 33 items questionnaire. The FLCAS was adopted and translated into Indonesian language to make the students respond easily. The participants of this research are 20 students in the $10^{\text {th }}$ grade at SMA PASUNDAN CIKALONG KULON Cianjur in academic year 2017/2018.

\section{RESULTS AND DISCUSSION}

\section{Results}

In this result, researchers describe and discuss about "student anxiety in learning speaking" using drill method. The Likert's scoring scale table to measure students' anxiety level using FLCAS by Horwitz, et al (1986) stated in (Mayangta, 2013) is shown in below. 
Table 1.Likert's scoring table stated in (Mayangta, 2013)

\begin{tabular}{cccccc}
\hline & \multicolumn{5}{c}{ Scoring } \\
\cline { 2 - 6 } Statement & $\begin{array}{c}\text { Strongly } \\
\text { Agree }\end{array}$ & Agree & $\begin{array}{c}\text { Neither } \\
\text { Agree nor } \\
\text { Disagree }\end{array}$ & Disagree & $\begin{array}{c}\text { Strongly } \\
\text { Disagree }\end{array}$ \\
\hline Positive & 1 & 2 & 3 & 4 & 5 \\
\hline Negative & 5 & 4 & 3 & 2 & 1 \\
\hline
\end{tabular}

As for the categorization of the anxiety level, the research used and adapted Oetting's scale which total score of 200. Based on table 1 . Most of the students who answered strongly agree in negative statement it means the students are relaxed, while if a little of students answered strongly agree in positive statement it means the students are anxious.

Table 2. FLCAS Anxiety Scale Adapted from Oetting'sScalestated in (Mayangta, 2013)

\begin{tabular}{cc}
\hline Range & Level \\
\hline $124-165$ & Very Anxious \\
$108-123$ & Anxious \\
$87-107$ & Mildly Anxious \\
$66-86$ & Relaxed \\
$33-65$ & Very Relaxed \\
\hline
\end{tabular}

After scoring, Researchers got the result of analyzing students' anxiety. In class X mipa 1 using drill method.

Table 3. The Result of Students Questionnaire Answer

\begin{tabular}{|c|c|c|c|c|c|c|c|c|c|c|c|c|c|c|c|c|c|c|c|c|c|c|c|c|c|c|c|c|c|c|c|c|c|c|}
\hline \multirow{2}{*}{ No } & \multirow{2}{*}{ Name } & \multicolumn{33}{|c|}{ Question Number } \\
\hline & & 1 & 2 & 3 & 4 & 5 & 6 & 1 & 8 & 9 & 10 & 11 & 12 & 13 & 14 & 15 & 16 & 17 & 18 & 19 & 20 & 21 & 22 & 23 & 24 & 25 & 26 & 27 & 28 & 29 & 30 & 31 & 32 & \\
\hline 1 & Students 1 & D & D & ND & A & ND & ND & SD & ND & D & D & ND & D & $\mathrm{ND}$ & $\mathrm{ND}$ & SD & D & D & ND & $\mathrm{ND}$ & D & D & $\mathrm{ND}$ & $\mathrm{ND}$ & D & D & $\mathrm{ND}$ & D & $\mathrm{ND}$ & D & D & ND & D & D \\
\hline 2 & 52 & D & D & ND & ND & ND & ND & SD & ND & ND & D & ND & $\mathrm{ND}$ & D & D & SD & ND & D & $\mathrm{ND}$ & $\mathrm{ND}$ & $\mathrm{ND}$ & ND & D & ND & A & ND & D & ND & D & D & ND & ND & D & D \\
\hline 3 & Students 3 & ND & $A$ & $\mathrm{ND}$ & D & D & A & SD & A & D & D & D & $\mathrm{ND}$ & $\mathrm{ND}$ & $\mathrm{ND}$ & SD & D & D & A & D & D & D & $\mathrm{ND}$ & $\mathrm{ND}$ & SA & $\mathrm{ND}$ & $\mathrm{ND}$ & D & $\mathrm{ND}$ & $\mathrm{ND}$ & ND & D & $\mathrm{ND}$ & SD \\
\hline 4 & Students 4 & D & $\mathrm{ND}$ & D & D & A & SD & D & SA & D & $\mathrm{ND}$ & D & D & D & D & D & D & $\mathrm{ND}$ & $\mathrm{ND}$ & D & D & D & $\mathrm{ND}$ & D & D & $\mathrm{ND}$ & $\mathrm{ND}$ & ND & $\mathrm{ND}$ & $\mathrm{ND}$ & $\mathrm{ND}$ & $\mathrm{ND}$ & $\mathrm{ND}$ & D \\
\hline 5 & Students 5 & D & D & $\mathrm{ND}$ & $\mathrm{ND}$ & SD & SD & $\mathrm{ND}$ & D & D & D & D & D & $S D$ & $S D$ & $\mathrm{ND}$ & D & D & A & D & D & D & $\mathrm{ND}$ & D & $\mathrm{ND}$ & D & $\mathrm{ND}$ & D & A & D & $\mathrm{ND}$ & D & $\mathrm{ND}$ & $\mathrm{ND}$ \\
\hline 6 & Students 6 & SD & D & D & D & SD & D & ND & D & $\mathrm{ND}$ & $\mathrm{ND}$ & D & D & SD & $S D$ & D & D & D & $\mathrm{ND}$ & $\mathrm{ND}$ & D & $\mathrm{ND}$ & $\mathrm{ND}$ & D & D & D & D & SD & A & D & D & D & $\mathrm{ND}$ & D \\
\hline 7 & 57 & SD & D & SD & SD & D & SD & SD & $\mathrm{ND}$ & SD & SD & D & SD & D & D & SD & SD & D & $\mathrm{ND}$ & D & SD & $\mathrm{SD}$ & $\mathrm{ND}$ & D & SD & D & $S D$ & D & A & SD & D & SD & D & D \\
\hline 8 & Students 8 & $\mathrm{ND}$ & D & $\mathrm{ND}$ & A & $\mathrm{ND}$ & SA & A & D & SA & $\mathrm{ND}$ & $S D$ & A & D & D & A & A & A & $\mathrm{ND}$ & $\mathrm{ND}$ & $\mathrm{ND}$ & A & $\mathrm{ND}$ & A & A & $\mathrm{ND}$ & A & A & SD & SA & A & A & $\mathrm{ND}$ & SA \\
\hline 9 & Students 9 & A & $\mathrm{SD}$ & A & SA & D & SA & SA & ND & A & A & D & A & $S D$ & SD & D & A & $\mathrm{SD}$ & D & A & D & $\mathrm{ND}$ & D & SA & SA & A & ND & A & D & $\mathrm{ND}$ & A & A & D & D \\
\hline 10 & 510 & SD & D & SD & SD & $\mathrm{ND}$ & SD & SD & SA & SD & SD & $\mathrm{ND}$ & $\mathrm{SD}$ & D & D & D & D & D & A & SD & D & D & $\mathrm{ND}$ & D & D & D & D & SD & $\mathrm{ND}$ & SA & D & SD & $\mathrm{ND}$ & SD \\
\hline 11 & Students 11 & $\mathrm{~A}$ & D & A & D & A & D & A & ND & A & A & D & D & $\mathrm{ND}$ & ND & D & ND & D & D & $\mathrm{ND}$ & A & A & $\mathrm{ND}$ & $\mathrm{ND}$ & A & A & ND & A & $\mathrm{ND}$ & $\mathrm{ND}$ & A & A & $\mathrm{ND}$ & A \\
\hline 12 & Students 12 & $2 \mathrm{D}$ & SD & D & $\mathrm{ND}$ & SD & $\mathrm{ND}$ & D & $\mathrm{ND}$ & $\mathrm{ND}$ & SA & $\mathrm{ND}$ & D & D & D & SD & D & $S D$ & $\mathrm{ND}$ & D & D & D & D & $S D$ & D & $\mathrm{ND}$ & SD & $\mathrm{ND}$ & D & $\mathrm{ND}$ & D & $\mathrm{ND}$ & $\mathrm{ND}$ & $\mathrm{ND}$ \\
\hline 13 & Stude & $\mathrm{NA}$ & SD & $\mathrm{ND}$ & D & D & D & D & $\mathrm{ND}$ & $\mathrm{ND}$ & D & D & $\mathrm{ND}$ & D & D & D & $\mathrm{ND}$ & D & D & D & D & D & $\mathrm{ND}$ & $S D$ & D & D & A & D & $\mathrm{ND}$ & D & D & D & A & D \\
\hline 14 & Students 14 & $4 \mathrm{D}$ & D & ND & $\mathrm{ND}$ & SD & A & A & A & ND & $\mathrm{ND}$ & D & D & D & D & A & A & $\mathrm{ND}$ & ND & A & $\mathrm{ND}$ & A & D & $\mathrm{ND}$ & $\mathrm{ND}$ & A & $\mathrm{ND}$ & $\mathrm{ND}$ & A & A & ND & D & $\mathrm{ND}$ & A \\
\hline 15 & Students 15 & D & SD & $\mathrm{ND}$ & D & D & D & ND & ND & D & ND & D & $\mathrm{ND}$ & SD & SD & ND & ND & A & ND & D & $\mathrm{ND}$ & $\mathrm{ND}$ & $\mathrm{ND}$ & D & D & ND & D & A & $\mathrm{ND}$ & SA & A & ND & D & SA \\
\hline 16 & Students 16 & 6 SD & SD & SD & SD & SD & SD & SD & A & $S D$ & D & ND & SD & D & D & D & D & $S D$ & D & $S D$ & $S D$ & SD & $\mathrm{ND}$ & D & D & SD & $S D$ & SD & D & SD & SD & SD & ND & $S D$ \\
\hline 17 & Students 17 & $\mathrm{D}$ & $\mathrm{ND}$ & D & A & SD & D & D & $\mathrm{ND}$ & D & $\mathrm{ND}$ & $\mathrm{ND}$ & SD & $\mathrm{ND}$ & $\mathrm{ND}$ & SD & D & D & D & D & $\mathrm{ND}$ & SD & $\mathrm{ND}$ & $\mathrm{ND}$ & D & $\mathrm{ND}$ & $\mathrm{A}$ & D & $\mathrm{ND}$ & D & A & D & A & D \\
\hline 18 & Students 18 & SD & D & D & SD & SD & SD & SD & A & SD & SD & ND & SD & D & D & SD & D & SD & D & D & SD & SD & $\mathrm{ND}$ & D & SD & D & D & SD & D & SD & SD & SD & $\mathrm{ND}$ & SD \\
\hline 19 & Students 19 & $9 \mathrm{SD}$ & D & D & SD & D & SD & SD & $\mathrm{ND}$ & D & $S D$ & $\mathrm{ND}$ & SD & D & D & D & D & SD & D & D & SD & $S D$ & $\mathrm{ND}$ & SD & D & SD & D & SD & SD & SD & SD & SD & SA & D \\
\hline 20 & Students 20 & & $\mathrm{ND}$ & D & D & A & ND & D & ND & ND & A & A & D & A & A & D & SD & SD & SD & D & $\mathrm{ND}$ & D & A & D & D & $\mathrm{ND}$ & $\mathrm{ND}$ & D & A & D & ND & ND & A & SD \\
\hline
\end{tabular}


Yellow Colums

Red Colums
: Positive Statements

: Negative Statements

By which :

SA : Strongly Agree

A : Agree

NA : Neither Agree nor Disagree

D : Disagree

SD : Strongly Disagree

Table 4. Result of analyzing students' anxiety using drill method.

\begin{tabular}{cc}
\hline Level & Percentage \\
\hline Very Anxious & $10 \%$ \\
Anxious & $15 \%$ \\
Mildly Anxious & $20 \%$ \\
Relaxed & $30 \%$ \\
Very Relaxed & $25 \%$ \\
\hline
\end{tabular}

From the table 4 . The result of analyzing students anxiety using drill method can described are $10 \%$ of students are very anxious, $15 \%$ of students are the pre anxious, $20 \%$ of students are mildly anxious, $30 \%$ of students are relaxed and $25 \%$ of students are very relaxed. It means drill is one of metho to solve the problem anxiety in learning speaking.

Table 5. The Percentage of Students' Preference

\begin{tabular}{|c|c|c|c|c|c|c|c|c|c|c|c|}
\hline \multirow{2}{*}{ No } & \multirow{2}{*}{ Questionaire } & \multicolumn{10}{|c|}{ The Percentage of Students' preference } \\
\hline & & $\mathbf{S A}$ & $\%$ & $\mathbf{A}$ & $\%$ & NA & $\%$ & D & $\%$ & SD & $\%$ \\
\hline 1 & $\begin{array}{l}\text { I never feel quite sure of } \\
\text { myself when I am speaking } \\
\text { in my foreign language } \\
\text { class. }\end{array}$ & $\mathbf{0}$ & $\mathbf{0}$ & 2 & $\begin{array}{l}10 \\
\%\end{array}$ & 3 & $\begin{array}{l}15 \\
\%\end{array}$ & 9 & $\begin{array}{l}45 \\
\%\end{array}$ & 6 & $\begin{array}{l}30 \\
\%\end{array}$ \\
\hline 2 & $\begin{array}{l}\text { I don't worry about making } \\
\text { mistakes in language class. }\end{array}$ & $\mathbf{0}$ & $\begin{array}{l}\mathbf{0} \\
\%\end{array}$ & 1 & $\begin{array}{l}5 \\
\%\end{array}$ & 3 & $\begin{array}{l}15 \\
\%\end{array}$ & 11 & $\begin{array}{l}55 \\
\%\end{array}$ & 5 & $\begin{array}{l}25 \\
\%\end{array}$ \\
\hline 3 & $\begin{array}{l}\text { I tremble when I know that } \\
\text { I'm going to be called on in } \\
\text { language class. }\end{array}$ & $\mathbf{0}$ & $\begin{array}{l}\mathbf{0} \\
\%\end{array}$ & 2 & $\begin{array}{l}10 \\
\%\end{array}$ & 8 & $\begin{array}{l}40 \\
\%\end{array}$ & 6 & $\begin{array}{l}30 \\
\%\end{array}$ & 4 & $\begin{array}{l}20 \\
\%\end{array}$ \\
\hline 4 & $\begin{array}{l}\text { It frightens me when I don't } \\
\text { understand what the teacher } \\
\text { is saying in the foreign } \\
\text { language. }\end{array}$ & 1 & $\begin{array}{l}5 \\
\%\end{array}$ & 3 & $\begin{array}{l}15 \\
\%\end{array}$ & 4 & $\begin{array}{l}20 \\
\%\end{array}$ & 7 & $\begin{array}{l}35 \\
\%\end{array}$ & 5 & $\begin{array}{l}25 \\
\%\end{array}$ \\
\hline 5 & $\begin{array}{l}\text { It wouldn't bother me at all } \\
\text { to take more foreign } \\
\text { language classes. }\end{array}$ & $\mathbf{0}$ & $\begin{array}{l}0 \\
\%\end{array}$ & 3 & $\begin{array}{l}15 \\
\%\end{array}$ & 4 & $\begin{array}{l}20 \\
\%\end{array}$ & 6 & $\begin{array}{l}30 \\
\%\end{array}$ & 7 & $\begin{array}{l}35 \\
\%\end{array}$ \\
\hline 6 & $\begin{array}{l}\text { During language class, I } \\
\text { find myself thinking about } \\
\text { things that have nothing to } \\
\text { do with the course. }\end{array}$ & 2 & $\begin{array}{l}10 \\
\%\end{array}$ & 2 & $\begin{array}{l}10 \\
\%\end{array}$ & 4 & $\begin{array}{l}20 \\
\%\end{array}$ & 5 & $\begin{array}{l}25 \\
\%\end{array}$ & 7 & $\begin{array}{l}35 \\
\%\end{array}$ \\
\hline
\end{tabular}




\begin{tabular}{|c|c|c|c|c|c|c|c|c|c|c|c|}
\hline 7 & $\begin{array}{l}\text { I keep thinking that the other } \\
\text { students are better at } \\
\text { languages than I am. }\end{array}$ & 1 & $\begin{array}{l}5 \\
\%\end{array}$ & 3 & $\begin{array}{l}15 \\
\%\end{array}$ & 3 & $\begin{array}{l}15 \\
\%\end{array}$ & 5 & $\begin{array}{l}25 \\
\%\end{array}$ & 8 & $\begin{array}{l}40 \\
\%\end{array}$ \\
\hline 8 & $\begin{array}{l}\text { I am usually at ease during } \\
\text { tests in my language class. }\end{array}$ & 2 & $\begin{array}{l}10 \\
\%\end{array}$ & 6 & $\begin{array}{l}30 \\
\%\end{array}$ & 11 & $\begin{array}{l}55 \\
\%\end{array}$ & 1 & $\begin{array}{l}5 \\
\%\end{array}$ & $\mathbf{0}$ & $\begin{array}{l}\mathbf{0} \\
\%\end{array}$ \\
\hline 9 & $\begin{array}{l}\text { I start to panic when I have } \\
\text { to speak without preparation } \\
\text { in language class. }\end{array}$ & 1 & $\begin{array}{l}5 \\
\%\end{array}$ & 2 & $\begin{array}{l}10 \\
\%\end{array}$ & 6 & $\begin{array}{l}30 \\
\%\end{array}$ & 7 & $\begin{array}{l}35 \\
\%\end{array}$ & 4 & $\begin{array}{l}20 \\
\%\end{array}$ \\
\hline 10 & $\begin{array}{l}\text { I worry about the } \\
\text { consequences of failing my } \\
\text { foreign language class. }\end{array}$ & 1 & $\begin{array}{l}5 \\
\%\end{array}$ & 3 & $\begin{array}{l}15 \\
\%\end{array}$ & 6 & $\begin{array}{l}30 \\
\%\end{array}$ & 6 & $\begin{array}{l}30 \\
\%\end{array}$ & 4 & $\begin{array}{l}20 \\
\%\end{array}$ \\
\hline 11 & $\begin{array}{l}\text { I don't understand why } \\
\text { some people get so upset } \\
\text { over foreign language } \\
\text { classes. }\end{array}$ & $\mathbf{0}$ & $\begin{array}{l}\mathbf{0} \\
\%\end{array}$ & 1 & $\begin{array}{l}5 \\
\%\end{array}$ & 8 & $\begin{array}{l}40 \\
\%\end{array}$ & 10 & $\begin{array}{l}50 \\
\%\end{array}$ & 1 & $\begin{array}{l}5 \\
\%\end{array}$ \\
\hline 12 & $\begin{array}{l}\text { In language class, I can get } \\
\text { so nervous I forget things I } \\
\text { know. }\end{array}$ & $\mathbf{0}$ & $\begin{array}{l}\text { 0 } \\
\%\end{array}$ & 2 & $\begin{array}{l}10 \\
\%\end{array}$ & 4 & $\begin{array}{l}20 \\
\%\end{array}$ & 8 & $\begin{array}{l}40 \\
\%\end{array}$ & 6 & $\begin{array}{l}30 \\
\%\end{array}$ \\
\hline 13 & $\begin{array}{l}\text { It embarrasses me to } \\
\text { volunteer answers in my } \\
\text { language class. }\end{array}$ & $\mathbf{0}$ & $\begin{array}{l}\mathbf{0} \\
\%\end{array}$ & 3 & $\begin{array}{l}15 \\
\%\end{array}$ & 6 & $\begin{array}{l}30 \\
\%\end{array}$ & 8 & $\begin{array}{l}40 \\
\%\end{array}$ & 3 & $\begin{array}{l}15 \\
\%\end{array}$ \\
\hline 14 & $\begin{array}{l}\text { I would not be nervous } \\
\text { speaking the foreign } \\
\text { language with native } \\
\text { speakers. }\end{array}$ & $\mathbf{0}$ & $\begin{array}{l}\mathbf{0} \\
\%\end{array}$ & 1 & $\begin{array}{l}5 \\
\%\end{array}$ & 4 & $\begin{array}{l}20 \\
\%\end{array}$ & 11 & $\begin{array}{l}55 \\
5\end{array}$ & 4 & $\begin{array}{l}20 \\
\%\end{array}$ \\
\hline 15 & $\begin{array}{l}\text { I get upset when I don't } \\
\text { understand what the teacher } \\
\text { is correcting. }\end{array}$ & $\mathbf{0}$ & $\begin{array}{l}\text { 0 } \\
\%\end{array}$ & 2 & $\begin{array}{l}10 \\
\%\end{array}$ & 2 & $\begin{array}{l}10 \\
\%\end{array}$ & 9 & $\begin{array}{l}45 \\
\%\end{array}$ & 7 & $\begin{array}{l}35 \\
\%\end{array}$ \\
\hline 16 & $\begin{array}{l}\text { Even if I am well prepared } \\
\text { for language class, I feel } \\
\text { anxious about it. }\end{array}$ & $\mathbf{0}$ & $\begin{array}{l}\mathbf{0} \\
\%\end{array}$ & 3 & $\begin{array}{l}15 \\
\%\end{array}$ & 4 & $\begin{array}{l}20 \\
\%\end{array}$ & 11 & $\begin{array}{l}55 \\
\%\end{array}$ & 2 & $\begin{array}{l}10 \\
\%\end{array}$ \\
\hline 17 & $\begin{array}{l}\text { I often feel like not going to } \\
\text { my language class. }\end{array}$ & $\mathbf{0}$ & $\begin{array}{l}\mathbf{0} \\
\%\end{array}$ & 2 & $\begin{array}{l}10 \\
\%\end{array}$ & 2 & $\begin{array}{l}10 \\
\%\end{array}$ & 10 & $\begin{array}{l}\mathbf{5 0} \\
\%\end{array}$ & 6 & $\begin{array}{l}30 \\
\%\end{array}$ \\
\hline 18 & $\begin{array}{l}\text { I feel confident when I } \\
\text { speak in foreign language } \\
\text { class. }\end{array}$ & 1 & $\begin{array}{l}5 \\
\%\end{array}$ & 1 & $\begin{array}{l}5 \\
\%\end{array}$ & 9 & $\begin{array}{l}45 \\
\%\end{array}$ & 9 & $\begin{array}{l}45 \\
\%\end{array}$ & $\mathbf{0}$ & $\begin{array}{l}\mathbf{0} \\
\%\end{array}$ \\
\hline 19 & $\begin{array}{l}\text { I am afraid that my language } \\
\text { teacher is ready to correct } \\
\text { every mistake I make. }\end{array}$ & $\mathbf{0}$ & $\begin{array}{l}\mathbf{0} \\
\%\end{array}$ & 2 & $\begin{array}{l}10 \\
\%\end{array}$ & 5 & $\begin{array}{l}25 \\
\%\end{array}$ & 11 & $\begin{array}{l}55 \\
\%\end{array}$ & 2 & $\begin{array}{l}10 \\
\%\end{array}$ \\
\hline 20 & $\begin{array}{l}\text { I can feel my heart } \\
\text { pounding when I'm going to } \\
\text { be called on in language } \\
\text { class. }\end{array}$ & $\mathbf{0}$ & $\begin{array}{l}\mathbf{0} \\
\%\end{array}$ & 1 & $\begin{array}{l}5 \\
\%\end{array}$ & 6 & $\begin{array}{l}35 \\
\%\end{array}$ & 9 & $\begin{array}{l}45 \\
\%\end{array}$ & 4 & $\begin{array}{l}20 \\
\%\end{array}$ \\
\hline 21 & $\begin{array}{l}\text { The more I study for a } \\
\text { language test, the more con- } \\
\text { fused I get. }\end{array}$ & $\mathbf{0}$ & $\begin{array}{l}\mathbf{0} \\
\%\end{array}$ & 3 & $\begin{array}{l}15 \\
\%\end{array}$ & 4 & $\begin{array}{l}20 \\
\%\end{array}$ & 8 & $\begin{array}{l}40 \\
\%\end{array}$ & 5 & $\begin{array}{l}25 \\
\%\end{array}$ \\
\hline 22 & $\begin{array}{l}\text { I don't feel pressure to } \\
\text { prepare very well for } \\
\text { language class. }\end{array}$ & $\mathbf{0}$ & $\begin{array}{l}\mathbf{0} \\
\%\end{array}$ & 1 & $\begin{array}{l}5 \\
\%\end{array}$ & 15 & $\begin{array}{l}75 \\
\%\end{array}$ & 4 & $\begin{array}{l}20 \\
\%\end{array}$ & $\mathbf{0}$ & $\begin{array}{l}\mathbf{0} \\
\%\end{array}$ \\
\hline
\end{tabular}


Volume 1, No. 5, September 2018 pp 600-607

\begin{tabular}{|c|c|c|c|c|c|c|c|c|c|c|c|}
\hline 23 & $\begin{array}{l}\text { I always feel that the other } \\
\text { students speak the foreign } \\
\text { language better than I do. }\end{array}$ & 1 & $\begin{array}{l}5 \\
\%\end{array}$ & 2 & $\begin{array}{l}10 \\
\%\end{array}$ & 6 & $\begin{array}{l}30 \\
\%\end{array}$ & 8 & $\begin{array}{l}40 \\
\%\end{array}$ & 3 & $\begin{array}{l}15 \\
\%\end{array}$ \\
\hline 24 & $\begin{array}{l}\text { I feel very self-conscious } \\
\text { about speaking the foreign } \\
\text { language in front of other } \\
\text { students. }\end{array}$ & 2 & $\begin{array}{l}10 \\
\%\end{array}$ & 3 & $\begin{array}{l}15 \\
\%\end{array}$ & 2 & $\begin{array}{l}10 \\
\%\end{array}$ & 11 & $\begin{array}{l}55 \\
\%\end{array}$ & 2 & $\begin{array}{l}10 \\
5\end{array}$ \\
\hline 25 & $\begin{array}{l}\text { Language class moves so } \\
\text { quickly I worry about } \\
\text { getting left behind. }\end{array}$ & $\mathbf{0}$ & $\begin{array}{l}\mathbf{0} \\
\%\end{array}$ & 3 & $\begin{array}{l}15 \\
\%\end{array}$ & 8 & $\begin{array}{l}40 \\
\%\end{array}$ & 7 & $\begin{array}{l}35 \\
\%\end{array}$ & 2 & $\begin{array}{l}10 \\
5\end{array}$ \\
\hline 26 & $\begin{array}{l}\text { I feel more tense and nervous } \\
\text { in my language class than in } \\
\text { my other classes. }\end{array}$ & $\mathbf{0}$ & $\begin{array}{l}\mathbf{0} \\
\%\end{array}$ & 2 & $\begin{array}{l}10 \\
\%\end{array}$ & 8 & $\begin{array}{l}40 \\
\%\end{array}$ & 7 & $\begin{array}{l}35 \\
\%\end{array}$ & 3 & $\begin{array}{l}15 \\
\%\end{array}$ \\
\hline 27 & $\begin{array}{l}\text { I get nervous and confused } \\
\text { when I am speaking in my } \\
\text { language class. }\end{array}$ & $\mathbf{0}$ & $\begin{array}{l}\mathbf{0} \\
\%\end{array}$ & 4 & $\begin{array}{l}20 \\
\%\end{array}$ & 4 & $\begin{array}{l}20 \\
\%\end{array}$ & 7 & $\begin{array}{l}35 \\
\%\end{array}$ & 5 & $\begin{array}{l}25 \\
\%\end{array}$ \\
\hline 28 & $\begin{array}{l}\text { When I'm on my way to } \\
\text { language class, I feel very } \\
\text { sure and relaxed. }\end{array}$ & $\mathbf{0}$ & $\begin{array}{l}\mathbf{0} \\
\%\end{array}$ & 5 & $\begin{array}{l}25 \\
\%\end{array}$ & 8 & $\begin{array}{l}40 \\
\%\end{array}$ & 5 & $\begin{array}{l}20 \\
\%\end{array}$ & 2 & $\begin{array}{l}10 \\
\%\end{array}$ \\
\hline 29 & $\begin{array}{l}\text { I get nervous when I don't } \\
\text { understand every word the } \\
\text { language teacher says. }\end{array}$ & 3 & $\begin{array}{l}15 \\
\%\end{array}$ & 1 & $\begin{array}{l}5 \\
\%\end{array}$ & 5 & $\begin{array}{l}25 \\
\%\end{array}$ & 7 & $\begin{array}{l}35 \\
\%\end{array}$ & 4 & $\begin{array}{l}20 \\
\%\end{array}$ \\
\hline 30 & $\begin{array}{l}\text { I feel overwhelmed by the } \\
\text { number of rules you have to } \\
\text { learn to speak a foreign } \\
\text { language. }\end{array}$ & 0 & $\begin{array}{l}\mathbf{0} \\
\%\end{array}$ & 5 & $\begin{array}{l}15 \\
\%\end{array}$ & 6 & $\begin{array}{l}30 \\
\%\end{array}$ & 6 & $\begin{array}{l}30 \\
\%\end{array}$ & 3 & $\begin{array}{l}15 \\
\%\end{array}$ \\
\hline 31 & $\begin{array}{l}\text { I am afraid that the other } \\
\text { students will laugh at me } \\
\text { when I speak the foreign } \\
\text { language. }\end{array}$ & $\mathbf{0}$ & $\begin{array}{l}\mathbf{0} \\
\%\end{array}$ & 3 & $\begin{array}{l}15 \\
\%\end{array}$ & 6 & $\begin{array}{l}30 \\
\%\end{array}$ & 6 & $\begin{array}{l}30 \\
\%\end{array}$ & 5 & $\begin{array}{l}25 \\
\%\end{array}$ \\
\hline 32 & $\begin{array}{l}\text { I would probably feel } \\
\text { comfortable around native } \\
\text { speakers of the foreign } \\
\text { language. }\end{array}$ & 1 & $\begin{array}{l}5 \\
\%\end{array}$ & 3 & $\begin{array}{l}15 \\
\%\end{array}$ & 11 & $\begin{array}{l}55 \\
\%\end{array}$ & 5 & $\begin{array}{l}25 \\
\%\end{array}$ & $\mathbf{0}$ & $\begin{array}{l}\mathbf{0} \\
\%\end{array}$ \\
\hline 33 & $\begin{array}{l}\text { I get nervous when the } \\
\text { language teacher asks } \\
\text { questions which I haven't } \\
\text { prepared in advance. }\end{array}$ & 2 & $\begin{array}{l}10 \\
\%\end{array}$ & 2 & $\begin{array}{l}10 \\
\%\end{array}$ & 2 & $\begin{array}{l}10 \\
\%\end{array}$ & 9 & $\begin{array}{l}45 \\
\%\end{array}$ & 5 & $\begin{array}{l}25 \\
\%\end{array}$ \\
\hline
\end{tabular}

From the table 5. There are 33 questions, consist of two kinds of questions which are positive and negative. Based on the table abovestrongly agree mostly answered by 3 students with percentage $15 \%$ in question number 29, agree mostly answered by 6 students in question number 8 with percentage 30\%, Neither agree mostly answered 15 students in question number 22 with percentage $75 \%$, disagree mostly answered by 11 students in question number 2,14 , $16,19,24$ with percentage $55 \%$, and strongly disagree mostly answered by 8 students in number 7 with percentage $40 \%$. 


\section{Discussion}

The researchers describe that the most of students are relaxed in learning speaking with percentage 30\% after using drill method. Before the researchers using drill method most of students are anxious, based on the teory of (Carlson and Buskist, 1997) say anxiety is "a sense of apprehension or doom that is accompanied by certain physiological reaction, such as accelerated heart rate, swety palm, and tightness in the stomach".

Therefore anxiety determines the students' perforformance in the classroom. Whereas drill method can help the students to reduce anxiety it is showed by the result that students were motivated to study the material and satisfied with the use of drill method. So the research showed that drill method can reduce anxiety in learning speaking.

\section{CONCLUSION}

Students surveyed in the present study demonstrate that speaking a foreign language in the class is a fairly stressfulactivity. A consistent number of them provesto be aware of the possible embarrassment experienced by other learners when they speak English as a foreign language, thus proving the importance which such a feeling has among language learners.

Based on the data the researchers can conclude that first is one of the factors of decreasing achievement of students is anxiety. Second, based on the research finding, learning with using drill method can decrease students' anxiety. The lastaccording to the data found, most of students are relaxed in learning English with using drill method. So, drill method can help the students in learning speaking.

\section{ACKNOWLEDGMENTS}

The first the researches would like to express thanks to the head master of SMA Pasundan Cikalongkulon, second to our lecturer who helped us in this reseaech as a guide. Third the researchers also thanks to the audiences at template in professional Journal of English Education (PROJECT) and IKIP Siliwangi supported received for the work being published.

\section{REFERENCES}

Carlson, N. R. and Buskist, W. (1997) Psychology: The Science of Behaviour. Needham Heights: viacom company.

Halgin, R. P. and Whitbourne, S. K. (2007) abnormal psychology: Clinical Perspectives on Psychological Dissorders. New York: McGraw-Hill.

Kartikasari, E., Zainal, A. and Salam, U. (2014) 'Improving Students' Speaking Ability Through Repetition Drill Technique', (1), pp. 1-13. Available at: http://download.portalgaruda.org/article.php?article=287610\&val=7230\&title=Improv ing Students Speaking Ability Through Drill Technique.

Kaswan \& Suprijadi, D. (2016) Research In English Language Education. Bandung: Putra Praktisi.

Matthews, A., Spratt, M. and Nelson, L. D. (1991) At the Chalkface: Practical Techniques in Language Teaching. UK: Thomas Nelson.

Mayangta, T. (2013) 'Students' Speaking Anxiety in an EFL Classroom', pp. 24-29. 
Ormrod, J. E. (2011) Educational Psychology: Developing Learner. Boston: Pearson Education.

Passer, M. W. et al. (2009) Psychology: The Science of Mind and Behavior. New York: McGraw-Hill.

Tornbury, S. (2005) How to teach speaking. England: Longmans.

Wahyuni, A. D. (2016) 'The effectivenes of short movie for teaching speaking'. 